\title{
Expression of CD90 and P75NTR stem cell markers in ameloblastomas: a possible role in their biological behavior
}

Fernanda Paula Yamamoto SILVA(a) Andrielle DIAS(b) Carolinne Almeida COELHO(b) Eliete Neves GUERRA(c) Ana Elizia Mascarenhas MARQUES(c) Daniel de Almeida DECURCIO(a) Andrea MANTESSO(d) Sérgio Elias Vieira CURY(e) Brunno Santos de Freitas SILVA(a)

(a) Universidade Federal de Goiás - UFG, Dental School, Department of Stomatology Sciences, Goiânia, GO, Brazil.

(b) Universidade Federal de Goiás - UFG, Dental School, Goiânia, GO, Brazil

(c) Universidade de Brasília - UnB, Health Sciences Faculty, Oral Histopathology Laboratory, Brasília, DF, Brazil.

(d) Universidade de São Paulo - USP, Dental School, Department of Oral Pathology, São Paulo, SP, Brazil.

(e)Centro Universitário de Volta Redonda - UNIFOA, Dental School, Department of Stomatology, Volta Redonda, RJ, Brazil

Declaration of Interests: The authors certify that they have no commercial or associative interest that represents a conflict of interest in connection with the manuscript.

\section{Corresponding Author:}

Brunno Santos de Freitas Silva

E-mail: brunno.santosfreitas@gmail.com

DOI: 10.1590/1807-3107BOR-2016.vol30.0109

Submitted: Jan 19, 2016

Accepted for publication: Jun 13, 2016

Last revision: Jul 25,2016
Abstract: Multicystic and unicystic ameloblastomas are benign odontogenic tumors that present distinct biological behavior. The investigation of stem cells has become an important branch of tumor biology, with several studies addressing the possible role of these cells in tumor growth, angiogenesis, progression, infiltration and invasiveness. This study evaluated the immunohistochemical expression of CD90(Thy-1) and P75NTR stem cell markers in multicystic and unicystic ameloblastomas. Seventeen (17) samples of ameloblastomas (multicystic, $\mathrm{n}=10$; unicystic, $\mathrm{n}=7$ ) were submitted to immunohistochemical reactions and graded semi-quantitatively. The Kolmogorov-Smirnov test was used to verify possible differences in CD90 and P75NTR expressions between multicystic and unicystic ameloblastomas $(\mathrm{p}<0.05)$. CD90 immunostaining was observed in all multicystic ameloblastoma specimens $(n=10)$, in the cytoplasm of the fibroblasts and vascular endothelial cells of the tumor stroma, near the neoplastic odontogenic epithelia. The staining of stromal CD90 was significantly higher in multicystic than in unicystic ameloblastomas ( $\mathrm{p}=0.003$ ). Nuclear P75NTR immunostaining was observed in all ameloblastoma specimens. A significant difference was seen in the epithelial staining of P75NTR between multicystic and unicystic types $(p=0.007)$. The increased expression of CD90 and P75NTR found in multicystic ameloblastomas suggests a behavioral biological difference between multicystic and unicystic ameloblastomas, as well as a difference in ameloblastoma development.

Keywords: Stem cells; Antigens, Thy-1; Ameloblastoma.

\section{Introduction}

Ameloblastoma is a benign but locally destructive odontogenic tumor associated with substantial morbidities ${ }^{1}$. Clinically, the ameloblastoma can be classified as multicystic, unicystic, peripheral and demosplastic ${ }^{2}$. Unicystic and multicystic ameloblastomas are the most prevalent types of ameloblastoma in the jaws, whereas the multicystic type is described as the most aggressive, with problematic recurrences ${ }^{3}$. In recent years, many studies have identified molecular alterations in the ameloblastoma ${ }^{4,5}$; however, its etiology and the causes of its persistent biologic behavior are still unclear.

According to the last WHO classification of odontogenic tumors, ameloblastomas are composed of a neoplastic odontogenic epithelium with 
mature fibrous stroma, without neoplastic odontogenic ectomesenchyme ${ }^{2}$. A substantial body of studies has demonstrated the role of the interactions between stroma and neoplastic cells in tumor biology $y^{6,7,8}$ as an important event in understanding the biological behavior of tumors.

There are few studies suggesting a regulatory role of the stromal cells in the mechanisms of local invasiveness of ameloblastomas ${ }^{9,10,11}$. This role is based on studies of epithelial mesenchymal transition promoters ${ }^{9,10}$ and cytokine secretion in stromal fibroblasts of ameloblatomas ${ }^{11}$.

In line with the tumor and stromal cell interaction theory, CD90 (Thy-1), a glycosylphosphatidylinositol (GPI)-anchored glycoprotein emerges as an important mesenchymal stem cell marker that plays a major role in tumor-stroma interactions, influencing the degree of invasiveness of several types of cancers ${ }^{6,7,8}$.

Recently, Juuri et al. ${ }^{12}$ suggested that ameloblastoma epithelial cells have some stem cell properties. According to their study, the expression of transcription factor SRY-related HMG-box gene 2(SOX2) in ameloblastomas is related to the maintenance of "stemness" in the tumor epithelial cells, and consequently influences its biological behavior. However, the authors assume that the analysis of a single marker is not sufficient to elucidate the molecular mechanism involved in ameloblastoma pathogenesis.

P75 neurotrophin receptor (P75NTR) is a low-affinity receptor for nerve growth factors (neurotrophins and pro-neurotrophins), widely studied because of its diverse biological responses in different pathways, including survival, migration and differentiation of normal and tumor epithelial stem cells. P75NTR is expressed in various types of stem cells and has been used to isolate stem cells with different maturation capacity ${ }^{13}$. Moreover, P75NTR seems to be deregulated in mutant ameloblasts, which may participate in the development of an odontogenic tumor of dental epithelium origin ${ }^{14}$.

Although there are some studies addressing the interaction between tumor-stromal cells and stem cell properties in ameloblastomas, there have been no studies that have evaluated CD90 and P75NTR expressions in ameloblastomas, or their biological behavior in relation to stem cell marker expression.
In this context, the aim of the present study was to evaluate the immunohistochemical expression of CD90 and P75NTR stem cell markers in multicystic and unicystic ameloblastoma specimens.

\section{Methodology}

\section{Study design}

Following approval of the study by the Human Ethics Committee of the Institutional Review Board, our specialized Oral Medicine and Oral Pathology Service searched for records of ameloblastoma cases between the years of 2004 and 2014. All available paraffin-embedded tissue samples were reviewed, and only the cases with satisfactory tissue and a confirmed diagnosis of multicystic or unicystic ameloblastomas were included in the sample. The clinical characteristics of 11 of the 17 selected cases of ameloblastoma were recorded, including gender, age, anatomic site, size (performed with radiographic exams using the CliniView $^{\mathrm{TM}}$ software) and radiographic characteristics.

\section{Immunohistochemistry}

Ten (10) samples of multicystic and seven (7) of unicystic ameloblastomas were submitted to $3 \mu \mathrm{m}$ histological sections followed by dewaxing and rehydration in a graded ethanol series. Antigen retrieval was performed by immersing the sections in a $10 \mathrm{mM}$ monohydrated citrate buffer solution ( $\mathrm{pH}$ 6.0), and heating them in a water bath at $95^{\circ} \mathrm{C}$ for 30 minutes. Internal peroxidase activity was inhibited by $6 \%$ hydrogen peroxide and a methanol solution. After washing with Tris buffer ( $\mathrm{pH}$ 7.4), the slides were incubated with anti-CD90 monoclonal antibody (Abcam, Cambridge, MA, USA, dilution 1:100) overnight at $4^{\circ} \mathrm{C}$, and with anti-P75NTR (Abcam, Cambridge, MA, US, dilution 1:100) for 30 minutes at room temperature. Omission of the specific primary antibody was used as the negative control, whereas breast carcinoma samples were used as the positive controls for both CD90 and P75NTR. The slides were subsequently exposed to avidin-biotin complex (LSAB-Kit + HRP; DakoCytomation, Carpinteria, CA, USA) and to 3,3'-diaminobenzidine chromogen (DAB+; DakoCytomation, Carpinteria, CA, USA), and were counterstained with Meyer's hematoxylin, dehydrated 
in ethanol, cleared in xylene, and mounted. Brown staining was considered as positive.

The immunohistochemical expression was analyzed in 4 randomly selected fields at 200x magnification and graded semi-quantitatively, based on a modified score $^{15}$, considering the percentage of positive cells: grade $0,0-1 \%$; grade $1,>1-10 \%$; grade $2,>10-25 \%$; grade $3,>25-75 \%$; and grade $4,>75-100 \%$ positive cells. CD90 positivity was determined in the cytoplasm of the fibroblasts and in the vascular endothelial cells of the tumor stroma. P75NTR positivity was determined in the nucleus of the odontogenic epithelial cells. Here we considered only the nuclear expression of P75NTR, because it seems that P75NTR in the nucleus plays a role in ameloblast differentiation ${ }^{14}$. The immunohistochemical reactivity was analyzed by two independent, blinded and calibrated observers, under light microscopy at 200-fold magnification.

\section{Statistical analysis}

The statistical analysis was performed by the Statistical Package for Social Sciences software (SPSS 16, Headquarters, Chicago, IL, USA). The KolmogorovSmirnov test was used to verify possible differences in CD90 and P75NTR expressions between multicystic and unicystic ameloblastomas. Spearman's correlation coefficient was performed to evaluate the correlation between CD90 and P75NTR expressions in multicystic and unicystic ameloblastomas. A $P$-value of $<0.05$ was considered statistically significant.

\section{Results}

\section{Clinical and pathological characteristics}

Clinical data were available for 11 of the 17 patients, 6 being male and 5 being female, with an average age of 30.36 years (range of $14-47$ years). Sixty-three percent $(63.64 \%-n=7)$ of the ameloblastomas were located in the posterior mandible and $36.36 \%(n=4)$ in the body of the mandible, with a median size of $78.71 \mathrm{~mm}$ (range of 45.3-127.4 mm). Radiographically, the most common features were well-defined uni- or multilocular radiolucencies, mainly affecting the mandible (Table 1).

\section{CD90 expression in multicystic and unicystic ameloblastomas}

CD90 immunostaining was observed in all the multicystic ameloblastoma specimens $(n=10)$, with a prominent cytoplasmic CD90 expression in the fibroblasts and the vascular endothelial cells of the tumor stroma, near the cords/nests of neoplastic odontogenic epithelia (Figure 1, A-B). Unicystic ameloblastomas showed discrete cytoplasmic CD90 immunostaining in the capsular connective tissue of the tumors, with no evident proximity to the neoplastic epithelium (Figure 1, C). The staining of stromal CD90 was significantly higher in multicystic ameloblastomas than in unicystic ameloblastomas (Kolmogorov-Smirnov test $-\mathrm{p}=0.003)$ (Table 2).

Table 1. Clinical and pathological features of the ameloblastoma sample.

\begin{tabular}{|c|c|c|c|c|c|c|}
\hline Case & Gender & Age (years) & Anatomic location & Pathological type & Radiographic characteristics & Size $(\mathrm{mm})$ \\
\hline 1 & M & 24 & Body of the mandible & plexiform & multilocular & 116,2 \\
\hline 2 & $\mathrm{~F}$ & 45 & Mandibular symphisis & plexiform & multilocular & 81,7 \\
\hline 3 & M & 47 & Posterior mandible & follicular & multilocular & 49,1 \\
\hline 4 & M & 35 & Posterior mandible & acantomathous & multilocular & 74,6 \\
\hline 5 & M & 22 & Mandibular symphisis & follicular & multilocular & 97,4 \\
\hline 6 & M & 41 & Body of the mandible & follicular & multilocular & 62,7 \\
\hline 7 & $\mathrm{~F}$ & 22 & Posterior mandible & luminal & unilocular & 45,3 \\
\hline 8 & $\mathrm{~F}$ & 20 & Posterior mandible & luminal & unilocular & 127,4 \\
\hline 9 & M & 14 & Posterior mandible & unspecified & unilocular & 59,9 \\
\hline 10 & $\mathrm{~F}$ & 19 & Body of the mandible & unspecified & unilocular & 66,1 \\
\hline 11 & $\mathrm{~F}$ & 45 & Posterior mandible & mural & unilocular & 85,5 \\
\hline
\end{tabular}



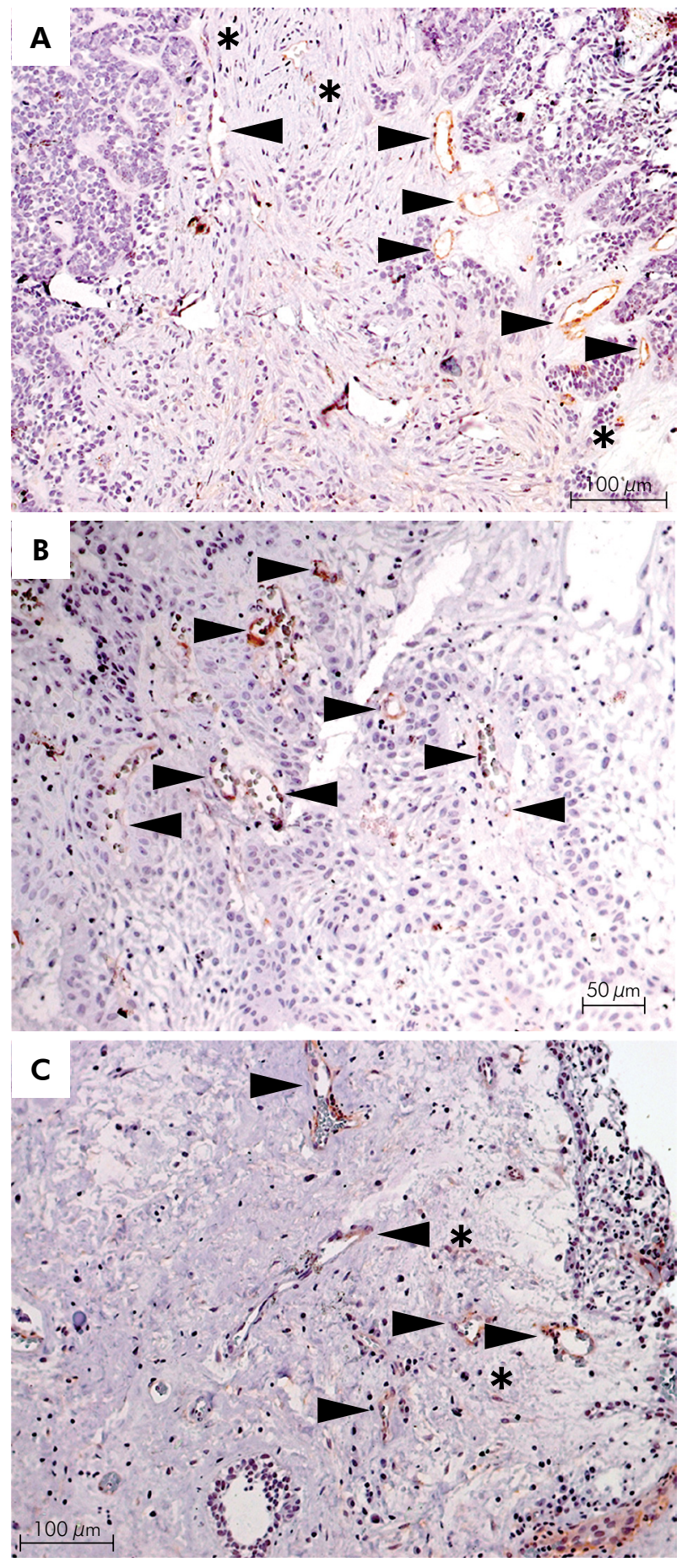

Figure 1. Immunohistochemical expression of CD90 in multicystic and unicystic ameloblastomas. (A, B) Cytoplasmic CD90 expression in the fibroblasts (asterisk) and vascular endothelial cells of the multicystic ameloblastoma tumor stroma, near the cords/nests of neoplastic odontogenic epithelia (arrows head). (C) Illustrates a weak cytoplasmic CD90 expression in a unicystic ameloblastoma in the capsular connective tissue of the tumor (fibroblasts - asterisk; vascular endothelial cells - arrows head). Magnification indicated in the scale bar.
Table 2. Diferences in CD90 expression in multicystic and unicystic ameloblastomas.*

\begin{tabular}{|c|c|c|c|c|c|}
\hline \multirow{2}{*}{$\begin{array}{l}\text { Score } \\
\text { (Grade) }\end{array}$} & \multicolumn{2}{|c|}{ Multicystic } & \multicolumn{2}{|c|}{ Unicystic } & \multirow{2}{*}{$\mathrm{p}$} \\
\hline & $\mathrm{n}$ & $\%$ & $n$ & $\%$ & \\
\hline 0 & 1 & 10 & 2 & 28,6 & - \\
\hline 1 & - & 0 & 5 & 71,4 & 0.003 \\
\hline 2 & 9 & 90 & - & 0 & - \\
\hline Total & 10 & 100 & 7 & 100 & - \\
\hline
\end{tabular}

*Kolmogorov Smirnov test.

\section{P75NTR expression in multicystic and unicystic ameloblastomas}

P75NTR immunostaining was observed in all multicystic and unicystic ameloblastoma specimens. In multicystic ameloblastomas, high nuclear P75NTR expression was detected in the central areas of neoplastic epithelium cords/nests, mostly in areas with loosely arranged cells, resembling the stellate reticulum (Figure 2, A), or in cells with squamous differentiation (Figure 2, B). P75NTR was also seen in the peripheral ameloblast-like columnar cells.

Nuclear P75NTR expression was noted in the neoplastic epithelium of a few specimens of unicystic ameloblastomas (Figure 2, C), insofar as a significant difference was seen in the staining between multicystic and unicystic types (Kolmogorov-Smirnov test $-\mathrm{P}=0.007$ ) (Table 3).

\section{Discussion}

Multicystic and unicystic ameloblastomas are benign odontogenic tumors that present distinct biological behavior. A comparison between the two histological subtypes in terms of biological behavior shows multicystic ameloblastoma as being more locally invasive, having worse prognosis and also having a greater tendency of recurrence ${ }^{1,16}$. On the other hand, unicystic lesions are the less aggressive type of ameloblastoma, with a better response to enucleation and curettage than the multicystic type $\mathrm{e}^{17}$.

The investigation of stem cells has become an important branch of tumor biology ${ }^{13}$, with several studies addressing the possible role of these cells 


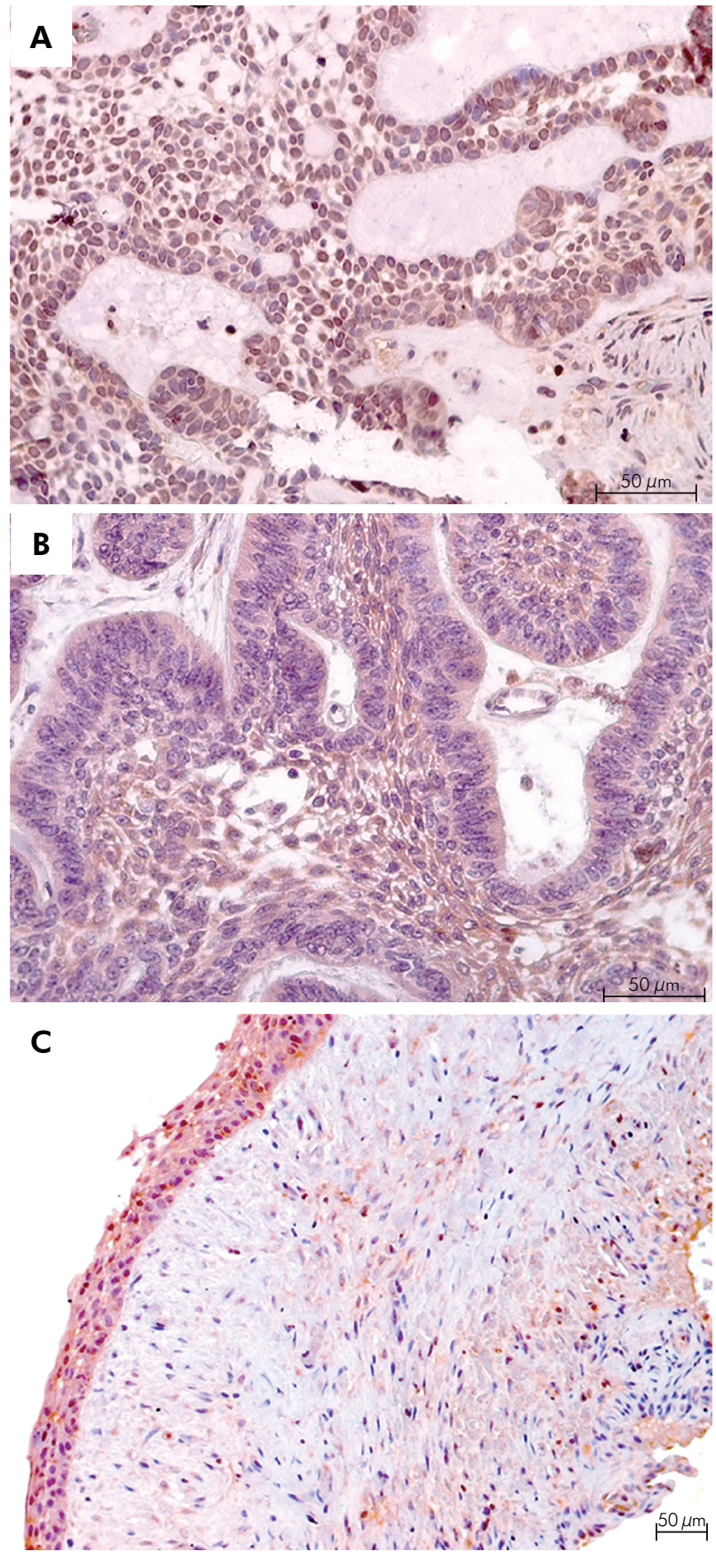

Figure 2. Immunohistochemical expression of P75NTR in multicystic and unicystic ameloblastomas. Nuclear P75NTR expression (A) in the peripheral ameloblast-like columnar cells and $(\mathrm{B})$ in central areas of neoplastic epithelium of a multicystic ameloblastoma. (C) Nuclear P75NTR expression in the neoplastic epithelium of a unicystic ameloblastoma. Magnification indicated in the scale bar.

in tumor growth, angiogenesis ${ }^{7}$, progression $^{18}$, infiltration and invasiviness ${ }^{19}$.

CD90 is an established marker for numerous types of human stem cells, especially mesenchymal
Table 3. Diferences in P75NTR expression in multicystic and unicystic ameloblastomas.*

\begin{tabular}{lcccccc}
\hline \multirow{2}{*}{$\begin{array}{l}\text { Score } \\
\text { (Grade) }\end{array}$} & \multicolumn{3}{c}{ Multicystic } & & \multicolumn{3}{c}{ Unicystic } & \\
\cline { 2 - 3 } & $n$ & $\%$ & & $n$ & $\%$ & $p$ \\
\hline 1 & 3 & 30 & & 7 & 100 & - \\
2 & 1 & 10 & & - & 0 & - \\
3 & 3 & 30 & & - & 0 & 0.007 \\
4 & 3 & 30 & & - & 0 & - \\
Total & 10 & 100 & & 7 & 100 & - \\
\hline
\end{tabular}

*Kolmogorov Smirnov test.

stem cells. It is considered a critical regulator of cell-cell and cell-matrix interactions, and plays a role in cellular migration and fibrosis, both events being considered important for tissue regeneration and oncogenesis ${ }^{20}$. CD90 expression in tumor stroma, mainly endothelial and mesenchymal stem cells, as well as in tumor-associated fibroblasts, seems to play an influential role in disease progression ${ }^{8}$.

In the present study, a significant CD90 expression was found in the stroma of all multicystic ameloblastoma specimens, whereas most unicystic cases showed a weak or nonexistent expression. Curiously, in multicystic ameloblastoma, CD90 staining was detected in the vascular endothelial cells and in the stromal fibroblasts near the neoplastic odontogenic epithelium. This finding highlights the potential of tumor stroma arising from an interaction with the neoplastic epithelium to mediate the invasive potential of multicystic ameloblastomas. This argument is based on the premise that when stroma lacks tumor block action, it may support tumor progression ${ }^{6}$. Additionally, it is known that tumor growth may be induced by the local microenvironment, which is an active phenomenon that occurs through the stromal cells (fibroblasts and vascular endothelium) and their tumor supporting role ${ }^{21}$.

The role of stromal cells has been recently highlighted in malignant and benign tumors, including ameloblastoma ${ }^{22}$. It has been theorized that the fibroblasts close to neoplastic cells can secrete inflammatory cytokines, which may induce 
osteoclastogenesis through the receptor activator of nuclear factor kappa B ligand (RANKL) ${ }^{23}$. Additionally, Sandra et al. ${ }^{24}$ showed that the ameloblastoma cells can secret RANKL and TNFa, which may induce osteoclastogenesis through stromal osteoclast activation.

Feng et al. ${ }^{9}$ suggested that the invasive potential of multicystic ameloblastoma could be regulated by interactions between the neoplastic and stromal cells, by regulating cellular adhesion and migration. Their theory was based on the expression of Twist, an epithelialmesenchymal transition (EMT) promoter in multicystic ameloblastomas, in stromal regions near the positive neoplastic cells. Furthermore, Siar and $\mathrm{Ng}^{10}$ found that ameloblastoma stromal fibroblasts and vascular endothelium were positive to Twist, proximal to positive neoplastic cells. We believe that CD90 expression in ameloblastoma, as in other tumors ${ }^{25}$, could play an important role in cellular adhesion and migration, serving as an important regulator of cell-matrix interactions ${ }^{7}$. Taking this into consideration, the prominent stromal CD90 expression in the multicystic (versus unicystic) ameloblastomas found in the present study could be one of the causes of the already known invasive behavior of the multicystic type.

In the present study we also analyzed the P75NTR expression in ameloblastoma specimens. Interestingly, a considerable expression of the P75NTR stem cell marker was detected in the central areas of the neoplastic epithelium, mostly in regions resembling the stellate reticulum or having squamous differentiation. P75NTR is a receptor for neurotrophins, and is related to pluripotency ${ }^{13}$. P75NTR has been found to be expressed in a variety of stem cell types, including embryonic ${ }^{26}$, adult and cancer stem cells ${ }^{15}$. It is also considered a stem cell marker of undifferentiated progenitors of the neural $\mathrm{crest}^{27}$. It is important to emphasize that the presence of P75NTR has been linked to a high rate of recurrence and aggressiveness of some tumors ${ }^{15}$. In addition, P75NTR is known as a nerve growth factor receptor marker ${ }^{15}$ and seems to participate in the fate of some events of neural crest cells, also involving the expression of the fibroblast growth factor (FGF) ${ }^{28,29}$. Recently, Nakao et al. ${ }^{30}$ suggested that FGF subtypes are involved in the proliferation of ameloblastomas. This could explain the increased P75NTR expression in the multicystic ameloblastomas presented here, and could also account for its distinct biological behavior in multicystic versus unicystic ameloblastomas.

The study results suggest that the expression of P75NTR could be related to ameloblastoma pathogenesis, since P75NTR is a marker of undifferentiated progenitors of the neural crest, which originates the odontoblasts ${ }^{13}$. Using an animal model, Fukumoto et al. (2004) $)^{14}$ showed that the expression of Msx2 and P75NTR in mutant tooth development is deregulated and increased, acquiring the properties of undifferentiated dental epithelium and resulting in the development of odontogenic tumors. In addition, Juuri et al. ${ }^{12}$ also suggested that stem cell properties are important in ameloblastoma formation and invasiveness, thus supporting the recurrent nature of some of these tumors. Considering this finding together with our results, we can postulate the possible role of P75NTR in the neoplastic conversion of the odontogenic epithelium present in the ameloblastoma.

\section{Conclusion}

To date, there have been no studies addressing the expression of CD90 and P75NTR stem cell markers in odontogenic tumors, especially in ameloblastoma. In this study, an increased expression of these two markers was found in multicystic ameloblastoma, thereby suggesting behavioral biological differences between multicystic and unicystic ameloblastomas, as well as a difference in ameloblastoma development, probably related to altered CD90 and P75NTR expressions.

\section{Acknowledgments}

The authors are grateful to Mr. Gercino Monteiro Filho for his assistance with the statistical analyses presented in the manuscript. The authors would also like to express their thanks to PIBIC/UFG/CNPq for their financial support. 


\section{References}

1. Chae MP, Smoll NR, Hunter-Smith DJ, Rozen WM.

Establishing the natural history and growth rate of ameloblastoma with implications for management: systematic review and meta-analysis. PLoS One. 2015;10(2):e0117241. doi:10.1371/journal.pone.0117241

2. Barnes L EJ, Reichart PA, Sidranskiy D. World Health Organization Classification of tumours, pathology and genetics of head and neck tumours. Lyon: IARC Press; 2005.

3. Antonoglou GN, Sándor GK. Recurrence rates of intraosseous ameloblastomas of the jaws: a systematic review of conservative versus aggressive treatment approaches and meta-analysis of non-randomized studies. J Craniomaxillofac Surg. 2015;43(1):149-57. doi:10.1016/j.jcms.2014.10.027

4. Kumamoto H, Izutsu T, Ohki K, Takahashi N, Ooya K. p53 gene status and expression of p53, MDM2, and p14 proteins in ameloblastomas. J Oral Pathol Med. 2004;33(5):292-9. doi:10.1111/j.0904-2512.2004.00044.x

5. Kumamoto H, Ohki K, Ooya K. Expression of Sonic hedgehog ( $\mathrm{SHH})$ signaling molecules in ameloblastomas. J Oral Pathol Med. 2004;33(3):185-90. doi:10.1111/j.0904-2512.2004.00070.x

6. Ampollini L, Madeddu D, Falco A, Frati C, Lorusso $B$, Graiani $G$ et al. Lung mesenchymal cells function as an inductive microenvironment for human lung cancer propagating cells. Eur J Cardiothorac Surg. 2014;46(6):e103-12. doi:10.1093/ejcts/ezu359

7. Zhu J, Thakolwiboon S, Liu X, Zhang M, Lubman DM. Overexpression of CD90 (Thy-1) in pancreatic adenocarcinoma present in the tumor microenvironment. PLoS One. 2014;9(12):e115507. doi:10.1371/journal.pone.0115507

8. Sukowati CH, Anfuso B, Crocé LS, Tiribelli C. The role of multipotent cancer associated fibroblasts in hepatocarcinogenesis. BMC Cancer. 2015;15(1):188. doi:10.1186/s12885-015-1196-y

9. Feng Y, Zhou YM, Hua CG, Tang XF, He DQ. Expression of Twist in different subtype of ameloblastomas. Oral Surg Oral Med Oral Pathol Oral Radiol Endod. 2009;108(4):565-70. doi:10.1016/j.tripleo.2009.05.041

10. Siar $\mathrm{CH}, \mathrm{Ng} \mathrm{KH}$. Differential expression of transcription factors Snail, Slug, SIP1, and Twist in ameloblastoma. J Oral Pathol Med. 2014;43(1):45-52. doi:10.1111/jop.12065

11. Fuchigami T, Kibe T, Hoyama H, Kishida S, Iijima M, Nishizawa $Y$ et al. Regulation of IL- 6 and IL-8 production by reciprocal cell-to-cell interactions between tumor cells and stromal fibroblasts through IL-1a in ameloblastoma Biochem Biophys Res Commun. 2014;451(4):491-6. doi:10.1016/j.bbrc.2014.07.137

12. Juuri E, Isaksson S, Jussila M, Heikinheimo K, Thesleff I. Expression of the stem cell marker, SOX2, in ameloblastoma and dental epithelium. Eur J Oral Sci. 2013;121(6):509-16. doi:10.1111/eos.12095
13. Tomellini E, Lagadec C, Polakowska R, Le Bourhis X. Role of p75 neurotrophin receptor in stem cell biology: more than just a marker. Cell Mol Life Sci. 2014;71(13):2467-81. doi:10.1007/s00018-014-1564-9

14. Fukumoto S, Kiba T, Hall B, Iehara N, Nakamura T, Longenecker $\mathrm{G}$, et al. Ameloblastin is a cell adhesion molecule required for maintaining the differentiation state of ameloblasts. J Cell Biol. 2004;167(5):973-83. doi:10.1083/jcb.200409077

15. Søland TM, Brusevold IJ, Koppang HS, Schenck K, Bryne $M$. Nerve growth factor receptor (p75 NTR) and pattern of invasion predict poor prognosis in oral squamous cell carcinoma. Histopathology. 2008;53(1):62-72. doi:10.1111/j.1365-2559.2008.03063.x

16. Reichart PA, Philipsen HP, Sonner S. Ameloblastoma: biological profile of 3677 cases. Eur J Cancer B Oral Oncol. 1995;31B(2):86-99. doi:10.1016/0964-1955(94)00037-5

17. Robinson L, Martinez MG. Unicystic ameloblastoma: a prognostically distinct entity. Cancer. 1977;40(5):2278-85. doi:10.1002/1097-0142(197711)40:5<2278::AID-CNCR282 0400539>3.0.CO;2-L

18. Hayashi S, Fujita K, Matsumoto S, Akita M, Satomi A. Isolation and identification of cancer stem cells from a side population of a human hepatoblastoma cell line, HuH-6 clone-5. Pediatr Surg Int. 2011;27(1):9-16. doi:10.1007/s00383-010-2719-x

19. Lee HJ, Noh KH, Lee YH, Song KH, Oh SJ, Kim SY, et al. NANOG signaling promotes metastatic capability of immunoedited tumor cells. Clin Exp Metastasis. 2015;32(5):429-39. doi:10.1007/s10585-015-9717-2

20. Rege TA, Hagood JS. Thy-1 as a regulator of cell-cell and cell-matrix interactions in axon regeneration, apoptosis, adhesion, migration, cancer, and fibrosis. FASEB J. 2006;20(8):1045-54. doi:10.1096/fj.05-5460rev

21. Tlsty TD, Coussens LM. Tumor stroma and regulation of cancer development. Annu Rev Pathol. 2006;1(1):119-50. doi:10.1146/annurev.pathol.1.110304.100224

22. Sathi GS, Nagatsuka H, Tamamura R, Fujii M, Gunduz M, Inoue M et al. Stromal cells promote bone invasion by suppressing bone formation in ameloblastoma. Histopathology. 2008;53(4):458-67. doi:10.1111/j.1365-2559.2008.03127.x

23. Takayanagi H, Iizuka H, Juji T, Nakagawa T, Yamamoto A, Miyazaki T et al. Involvement of receptor activator of nuclear factor kappaB ligand/osteoclast differentiation factor in osteoclastogenesis from synoviocytes in rheumatoid arthritis. Arthritis Rheum. 2000;43(2):259-69. doi:10.1002/15290131(200002)43:2<259::AID-ANR4>3.0.CO;2-W

24. Sandra F, Hendarmin L, Kukita T, Nakao Y, Nakamura N, Nakamura S. Ameloblastoma induces osteoclastogenesis: a possible role of ameloblastoma in expanding in the bone. Oral Oncol. 2005;41(6):637-44. doi:10.1016/j.oraloncology.2005.02.008 
25. Yang ZF, Ho DW, Ng MN, Lau CK, Yu WC, Ngai

$P$ et al. Significance of CD90+ cancer stem cells in human liver cancer. Cancer Cell. 2008;13(2):153-66. doi:10.1016/j.ccr.2008.01.013

26. Schuldiner M, Yanuka O, Itskovitz-Eldor J, Melton DA, Benvenisty N. Effects of eight growth factors on the differentiation of cells derived from human embryonic stem cells. Proc Natl Acad Sci USA. 2000;97(21):11307-12. doi:10.1073/pnas.97.21.11307

27. Fang D, Nguyen TK, Leishear K, Finko R, Kulp AN, Hotz $\mathrm{S}$, et al. A tumorigenic subpopulation with stem cell properties in melanomas. Cancer Res. 2005;65(20):9328-37. doi:10.1158/0008-5472.CAN-05-1343
28. Jiang X, Gwye Y, McKeown SJ, Bronner-Fraser M, Lutzko C, Lawlor ER. Isolation and characterization of neural crest stem cells derived from in vitro-differentiated human embryonic stem cells. Stem Cells Dev. 2009;18(7):1059-70. doi:10.1089/scd.2008.0362

29. Sieber-Blum M. Growth factor synergism and antagonism in early neural crest development. Biochem Cell Biol. 1998;76(6):1039-50. doi:10.1139/o99-016

30. Nakao Y, Mitsuyasu T, Kawano S, Nakamura N, Kanda S, Nakamura S. Fibroblast growth factors 7 and 10 are involved in ameloblastoma proliferation via the mitogen-activated protein kinase pathway. Int J Oncol. 2013;43(5):1377-84. doi:10.3892/ijo.2013.2081 